Nonlinear Processes in Geophysics (2002) 9: 75-78

Nonlinear Processes

in Geophysics

(C)European Geophysical Society 2002

\title{
A universal mirror wave-mode threshold condition for non-thermal space plasma environments
}

\author{
M. P. Leubner and N. Schupfer \\ Institute for Theoretical Physics, University of Innsbruck, Austria \\ Received: 11 July 2001 - Accepted: 16 October 2001
}

\begin{abstract}
Magnetic fluctuations are recognized in a large variety of space plasmas by increasingly high resolution, in situ observations as mirror wave mode structures. A typical requirement for the excitation of mirror modes is a dominant perpendicular pressure in a high-beta plasma environment. Contrary, we demonstrate from a realistic kinetic analysis how details of the velocity space distributions are of considerable significance for the instability threshold. Introducing the most common characteristics of observed ion and electron distributions by a mixed suprathermal-loss-cone, we derive a universal mirror instability criterion from an energy principle for collisionless plasmas. As a result, the transition from two temperature Maxwellians to realistic non-thermal features provides a strong source for the generation of mirror wave mode activity, reducing drastically the instability threshold. In particular, a number of space-related examples illuminate how the specific structure of the velocity space distribution dominates as a regulating excitation mechanism over the effects related to changes in the plasma parameters.
\end{abstract}

\section{Introduction}

Magnetic fluctuations are recognized in a large variety of space plasma environments by increasingly high resolution, in situ observations as mirror wave modes. Magnetohydrodynamic structures in the terrestrial magnetosheath were associated with the drift mirror instability (Pokhotelov and Pilipenko, 1976; Tsurutani et al., 1982) and ISEE 1 and 2 detections of mirror type modes downstream of the Earth's bow shock were reported (Hubert et al., 1989). The dispersion properties of $T_{\perp} / T_{\|}>1$ temperature anisotropy instabilities were discussed from kinetic aspects (McKean et al., 1992; Gary, 1992; Southwood and Kivelson, 1993; Kivelson, 1996) and illuminated by nonlinear effects (Pantellini, 1998). Recently, a study of the drift mirror instability in linear theory

Correspondence to: M. Leubner

(manfred.leubner@uibk.ac.at) for a high-beta single and two ion component space plasmas was provided by Pokhotelova (2001a, b).

Besides near Earth space, mirror waves have been systematically observed by Voyager 1 and 2 in Jupiter's and Saturn's magnetosheath, where, in particular, mirror mode structures were identified continuously from the bow shock down to the magnetopause. On the other hand, the identification of mirror mode structures in the solar wind were reported (Tsurutani et al., 1993) and solar wind magnetic holes are believed to result from mirror mode instability. Finally, high resolution VEGA or Giotto magnetic field measurements also confirm mirror wave generation driven by pressure anisotropies in the magnetospheres of comets (Glassmeier et al., 1993).

Typically, mirror wave modes are excited in high-beta plasmas where a significant pressure anisotropy $p_{\perp} / p_{\|}>1$ is present. Treating the hot plasma mirror instability in the MHD limit (Hastie and Taylor, 1964) is an assumption of fundamental criticism and a kinetic treatment of mirror wave mode excitation was already proposed by Tajiri (1967). Since suprathermal particle populations are known to be ubiquitous property of space plasma velocity distributions, recently, mirror wave mode excitation was studied in kinetic theory for kappa-distributions by Leubner and Schupfer (2000).

The generation of velocity space distributions exhibiting pronounced energetic tails are usually interpreted as a consequence of acceleration mechanisms (Gomberoff et al., 1995; Leubner, 2000a) and are known to be accurately represented by the family of kappa-distributions (Christon et al., 1988, 1991; Leubner, 2000b), introduced first by Vasyliunas (1968) and also related on observational grounds to solar wind electrons (Scudder and Olbert, 1979). Justification for the use of power-law distributions in space plasma modelling was provided (Hasegawa, 1985; Ma and Summers, 1998; Leubner, 2000a), and Treumann (1999a, b) demonstrated that $\kappa$-distributions are a particular thermodynamic equilibrium state. On the other hand, observed non-thermal features of velocity distributions related to loss-cone populations are inherent in any mirror configuration as the terrestrial magne- 
tosphere (Summers and Thorne, 1995) and are commonly modeled by a Dory-Guest-Harris type (DGH) distribution (Dory et al., 1965). In conjunction, we also mention the unique magnetic field of Jupiter's moon Ganymede as an example of creating a large loss-cone in the distribution of all charged particle populations (Williams and Mauk, 1997).

The following discussion of mirror instability thresholds is based on an analytical generalization of kappa-distributions to a mixed kappa-loss-cone velocity space representation, allowing one to study uniquely any kind of kinetic plasma processes based on the entire class of distributions ranging from two temperature Maxwellians to complex anisotropic suprathermal loss-cone structures.

\section{Theory}

We consider the ion velocity space distribution function $f(K, M)$ in terms of the kinetic energy $K=\frac{m}{2}\left(v_{\|}^{2}+v_{\perp}^{2}\right)$ and the magnetic moment $M=m v_{\perp}^{2} / 2 B$, where $v_{\|, \perp}$ are the velocity components parallel and perpendicular to the magnetic field $\boldsymbol{B}$, and $m$ is the mass of the species considered. Applying an energy principle for a collisionless plasma with pressures $p_{\|, \perp}$ yields the mirror instability condition in terms of the particle equilibrium distribution (Northrop and Schardt, 1980) as

$2 \pi \int_{0}^{\infty} d K \int_{0}^{K / B} \frac{M^{2} B^{3} d M}{\sqrt{2 m^{3}(K-M B)}}\left[-\frac{\partial f}{\partial K}\right]-p_{\perp}>\frac{B^{2}}{8 \pi}$.

In search of a highly general distribution function that generates with a minimum of free parameters, a large class of representations for astrophysical plasma applications, we introduce the family of kappa-distributions and model the ubiquitously observed suprathermal populations by a power law in particle speed. Furthermore, a large variety of space plasma environments as magnetospheric magnetic field configurations cause, in addition, loss-cone type velocity space distributions, characterized by $\partial f / \partial v>0$ for a certain range of particle speeds. To study the effect of the loss-cone property on plasma dynamics different loss-cone type distributions were introduced for space plasma modeling, as substracted Maxwellians or the DGH-type distribution.

We consider now the most general case of a loss-cone distribution occupied by a suprathermal particle population and combine the Maxwellian-type DGH distribution (Dory et al., 1965 ) with a two temperature power law in particle speed modeled by the family of kappa-distributions Leubner (1982) to obtain

$$
f_{\kappa j}\left(v_{\|}, v_{\perp}\right)=A\left(\frac{v_{\perp}}{v_{t h \perp}}\right)^{2 j}\left[1+\frac{v_{\|}^{2}}{\kappa v_{t h \|}^{2}}+\frac{v_{\perp}^{2}}{\kappa v_{t h \perp}^{2}}\right]^{-(\kappa+1)}
$$

where the normalization is provided for any value of the spectral index $\kappa$ and loss-cone index $j$ by

$$
A=\frac{N}{\pi^{3 / 2} v_{t h \|} v_{t h \perp}^{2}} \frac{\Gamma(\kappa+1)}{\kappa^{j+3 / 2} \Gamma(j+1) \Gamma(\kappa-j-1 / 2)} .
$$

Here, the parameter $\kappa$ shapes predominantly the suprathermal tails of this distribution, and $j$ is a measure of the loss-cone strength. The quantity $\Gamma$ denotes the standard Gamma function; $v_{t h \|, \perp}=\left(2 k_{B} T_{\|, \perp} / m\right)^{1 / 2}$ are the thermal speeds parallel and perpendicular to the magnetic field where the normalization is performed with respect to the particle density $N$. It was demonstrated by Leubner and Schupfer (2000) that suprathermal particle populations significantly reduce the mirror instability threshold, thus serving as a source of free energy.

The mixed loss-cone high energy-tail distribution (2), along with the normalization condition (3), reduces for $j=0$ to the family of kappa-distributions and for $\kappa \rightarrow \infty$ to the DGH distribution, whereas for both $j=0$ and $\kappa \rightarrow \infty$, the two temperature Maxwellian

$f_{M}\left(v_{\|}, v_{\perp}\right)=\frac{N}{\pi^{3 / 2} v_{t h \|} v_{t h \perp}^{2}} \exp \left[-\frac{v_{\|}^{2}}{v_{t h \|}^{2}}-\frac{v_{\perp}^{2}}{v_{t h \perp}^{2}}\right]$

is reproduced. Hence, the distribution function (2) provides a highly general velocity space model. This is applicable within the full range of space plasma observations that are known to stretch from isotropic and two temperature Maxwellians to a loss-cone type distribution or suprathermal particle populations, and as far as to loss-cone structures filled with a high energy plasma component. Hence, the velocity space distribution (2) is of considerable generality and importance, since it represents an analytical model applicable for most or, at least, a significantly large variety of space plasma environments observed.

Following Leubner and Schupfer (2001), the general case of a suprathermal particle population $(\kappa)$ embedded in a losscone structure $(j)$ is analyzed with respect to kinetic modifications of the mirror mode instability thresholds. Inserting the distribution function (2) into the kinetic instability criterion (1) and performing the integration in parallel and perpendicular velocity space, a solution $I(\kappa, j)$ for the integral on the left-hand side of Eq. (1) is obtained as

$I(j, \kappa)=\frac{N m}{4} \frac{v_{t h \perp}^{4}}{v_{t h \|}^{2}} \kappa \frac{(j+2)(j+1)}{\kappa-j-3 / 2}$.

Analytical expressions for the parallel and perpendicular pressures are found from the moments of the distribution function

$p_{\|}=\frac{N m}{2} v_{t h \|}^{2} \frac{\kappa}{\kappa-j-3 / 2}$

and

$p_{\perp}=\frac{N m}{2} v_{t h \perp}^{2} \frac{\kappa(j+1)}{\kappa-j-3 / 2}$,

providing, via the thermal velocities, the link to effective temperatures, commonly specified for $j=0$ in relation to the kappa-distributions (Leubner, 2000a). The integrals leading to Eqs. (5), (6) and (7) exist only for $\kappa-j-3 / 2>0$, thus generating a profound constraint where the energy of the distribution tends to infinity. 
(a)

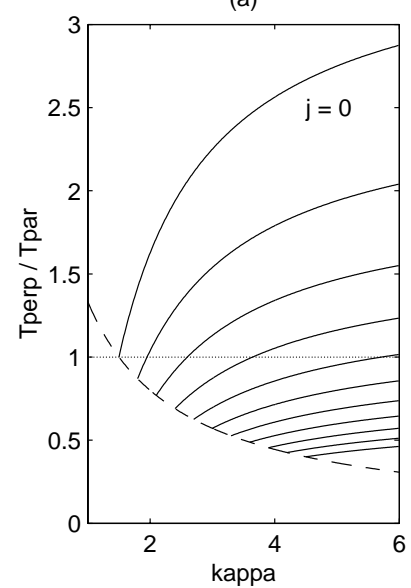

(b)

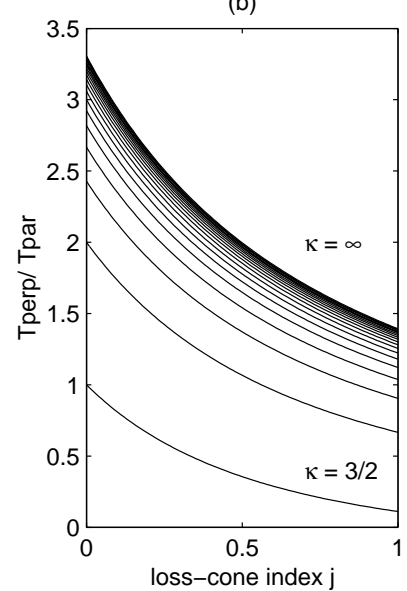

Fig. 1. Mirror instability thresholds for $\beta_{\perp}=0.4$ : (a) in the $T_{\perp} / T_{\|}-\kappa$ parameter space and values of the loss-cone index increasing by 0.3 from top $j=0$ to bottom $j=3$. The dashed line corresponds to the limit of the solution, see text. (b) In the $T_{\perp} / T_{\|}-j$ parameter space and values of $\kappa$ increasing by 1 from bottom $\kappa=3 / 2$ to top $\kappa=\infty$.

Finally, let $\beta_{\perp}=8 \pi N k_{B} T_{\perp} / B^{2}$ denote the standard (Maxwellian) beta. We rewrite condition (1) with respect to Eq. (5) and relation (7) in order to obtain a kinetic mirror instability criterion for mixed suprathermal loss-cone conditions as

$\beta_{\perp} \frac{\kappa(j+1)}{\kappa-j-3 / 2}\left[\frac{T_{\perp}}{T_{\|}} \frac{j+2}{2}-1\right]-1>0$

and the standard MHD limit $\beta_{\perp}\left(p_{\perp} / p_{\|}-1\right)-1>0$ for a two temperature Maxwellian distribution is recovered as $\kappa \rightarrow \infty$ and $j=0$. The mirror wave-mode threshold properties are found upon variation of the spectral index $\kappa$ and the loss-cone index $j$, both modeling details of the velocity space distribution function structure, along with the specific plasma parameters $\beta_{\perp}$ and the temperature or pressure anisotropy, respectively, of the space plasma environment considered.

\section{Results and conclusions}

The general case of combining both suprathermal and losscone effects in one distribution provides a concept applicable to a large class of plasma dynamics and related instability analysis. Let us study threshold situations not available from Leubner and Schupfer (2001), but of significance for a variety of magnetospheric and solar wind conditions.

Equating (8) to zero and solving for the temperature anisotropy, Fig. 1a shows the $\kappa$-dependent instability threshold for mirror wave-mode excitation as a function of $T_{\perp} / T_{\|}$ for discrete values of the loss-cone index between $0<j<3$. The presence of a significant fraction of energetic particles is the source for the generation of unstable and growing mirror modes due to an instability threshold reduction, an effect appearing further that is drastically increased for a nonzero loss-cone index. It turns out that the mirror wave-mode
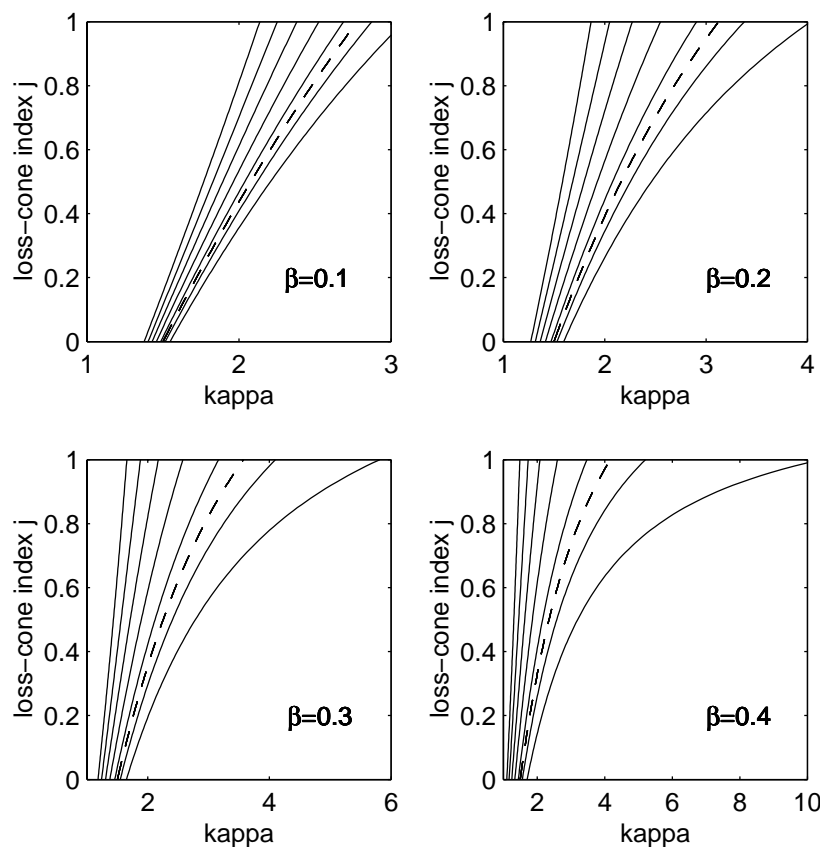

Fig. 2. Mirror instability thresholds in the $j-\kappa$ parameter space. The curves in each panel correspond in increasing manner from left to right to $T_{\perp} / T_{\|}=0.1 \ldots 1.3$ and the dashed line provides the case $T_{\perp} / T_{\|}=1$.

excitation is permissible for a certain range of small $\kappa$ values, even if $T_{\|}>T_{\perp}$, where the energy driving the effective perpendicular pressure originates from a suprathermal losscone ion population. In particular, a loss-cone index $j>1$ results in a shift of the instability threshold below temperature ratios $T_{\perp} / T_{\|} \sim 1$ for any value of the spectral index $\kappa$. Moreover, as already mentioned, the integration requires that $\kappa-j-3 / 2>0$ for the criterion to exist, wherefore the instability threshold cutoff is shifted simultaneously for increasing loss-cone indices to higher spectral indices $\kappa$, indicated by the dashed line. Figure $1 \mathrm{~b}$ provides instability thresholds for $T_{\perp} / T_{\|}$and a smooth increase in the loss-cone index $j$. Here, $\kappa$ serves as a parameter approaching from $\kappa=3 / 2$ the Maxwellian limit in the dark region where $\kappa=\infty$. Finally, Fig. 2 demonstrates for fixed $\beta_{\perp}$ the variation of the threshold, depending on the distribution function that shapes the parameters $\kappa$ and $j$ for some discrete values of the temperature anisotropy. The intersection between stability and mirror wave-mode excitation is found by equating condition (8) to zero and solving for $\kappa$. This yields

$\kappa=\frac{(j+3 / 2)}{1-\beta_{\perp}(j+1)\left[(j+2) T_{\perp} / 2 T_{\|}-1\right]}$.

The four panels clearly illuminate how the loss-cone strength and suprathermal populations dramatically reduce the mirror instability threshold, since the parameter space left of each curve corresponds to the mirror wave-mode growth. The dashed lines separate the left region, where $T_{\perp} / T_{\|}<1$, from those exceeding unity on the right and depicts the phys- 
ical situation where details of the distribution function significantly modify predictions of the MHD approximation.

We have demonstrated that the transition from a two temperature Maxwellian plasma to suprathermal loss-cone conditions provides a strong source for the generation of mirror wave-mode excitation, where the instability threshold is drastically reduced yielding wave mode activity even in the low- $\beta_{\perp}$ limit and for $T_{\|}>T_{\perp}$ for specific values of $\kappa$ and $j$. For non-thermal plasma characteristics, the particular structure of the velocity space distribution modeled by the spectral index $\kappa$ and loss-cone index $j$ dominates as a regulating mechanism for the mirror instability threshold over the effects related to changes in the plasma parameters $\beta_{\perp}$ and $T_{\perp} / T_{\|}$.

\section{References}

Christon, S. P., Mitchell, D. G., Williams, D. J., Frank, L. A., Huang, C. Y., and Eastman, T. E.: Energy spectra of plasma sheet ions and electrons from $\sim 50 \mathrm{eV} / \mathrm{e}$ to $\sim 1 \mathrm{MeV}$ during plasma temperature transitions, J. Geophys. Res., 93, 2562, 1988.

Christon, S. P., Williams, D. J., Mitchell, D. G., Huang, C. Y., and Frank, L. A.:, Spectral characteristics of plasma sheet ion and electron populations during disturbed geomagnetic conditions, J. Geophys. Res., 96, 1, 1991.

Dory, R. A., Guest, G. E., and Harris, E. G.: Unstable electrostatic plasma waves propagating perpendicular to a magnetic field, Phys. Rev. Lett., 14, 131, 1965.

Gary, S. P.: The mirror and ion cyclotron anisotropy instabilities, J. Geophys. Res., 97, 8519, 1992.

Glassmeier, K. H., Motschmann, U., Mazelle, C., Neubauer, F. M., Sauer, K., Fuselier, S. A., and Acuña, H.: Mirror modes and fast magnetoacoustic waves near the magnetic pileup boundary of comet P/Halley, J. Geophys. Res., 98, 20955, 1993.

Gomberoff, L., Hernandez, R., and Livi, S.: High energy tail formation due to right-hand polarized ion cyclotron waves, Planet. Space Sci., 43, 1101, 1995.

Hasegawa, A., Mima, K., and Duong-van M.: Plasma distribution function in a superthermal radiation field, Phys. Rev. Lett., 54, $2608,1985$.

Hastie, R. J. and Taylor, J. B.: Stability of magnetic wells at finite plasma pressure, Phys. Rev. Lett., 13, 123, 1964.

Hubert, D., Perche, C., Harvey, C. C., Lacombe, C., and Russell, C. T.: Observation of mirror waves downstream of a quasiperpendicular shock, Geophys. Res. Lett., 16, 159, 1989.

Kivelson, M. G. and Southwood, D. J.: Mirror instability II. The mechanism of nonlinear saturation, J. Geophys. Res., 101, 17365, 1996.

Leubner, M. P.: On Jupiter's whistler emission, J. Geophys. Res., 87, 6335, 1982.

Leubner, M. P.: Wave induced suprathermal tail generation of electron velocity space distributions, Planet. Space Sci., 48, 133, 2000a.

Leubner, M. P.: Theoretical interpretation of Jupiter's multibanded whistler mode emission, J. Geophys. Res., 105, 21 261, 2000b.

Leubner, M. P. and Schupfer, N.: Mirror instability thresholds in suprathermal space plasmas, J. Geophys. Res., 105, 27387 , 2000.

Leubner, M. P., and Schupfer, N.: A general kinetic mirror instability criterion for space applications, J. Geophys. Res., 106, 12993, 2001.

Ma, C. and Summers, D.: Formation of power-law energy spectra in space plasmas by stochastic acceleration due to whistler-mode waves, Geophys. Res. Lett., 25, 4099, 1998.

McKean, M. E., Winske, D., and Gary, S. P.: Mirror and ion cyclotron anisotropy instabilities in the magnetosheath, J. Geophys. Res., 97, 19421, 1992.

Northrop, T. G. and Schardt, A. W.: Instability of equatorial protons in Jupiter's mid-magnetosphere, J. Geophys. Res., 85, 25, 1980.

Pantellini, F. G. E.: A model of the formation of stable nonpropagating magnetic structures in the solar wind based on the nonlinear mirror instability, J. Geophys. Res., 103, 4789, 1998.

Pokhotelov, O. A. and Pilipenko, V. A.: Contributions to the theory of the drift-mirror instability of the magnetospheric plasma, Geomagn. Aeron., 16, 296, 1976.

Pokhotelov, O. A., Balikhin, M. A., Treumann, R. A., and Pavlenko, V. P.: Drift mirror instability revisted, 1: Cold electron temperature limit, J. Geophys. Res, 106, 8455, 2001a.

Pokhotelov, O. A., Onishchenko, O. G., Balikhin, M. A., Treumann, R. A., and Pavlenko, V. P.: Drift mirror instability in space plasmas, 2: Nonzero electron temperature effects, J. Geophys. Res, $106,13237,2001 \mathrm{~b}$

Scudder, J. D. and Olbert, S.: A theory of local and global processes which affect solar wind electrons. I. The origin of typical $1 \mathrm{AU}$ velocity distribution functions - steady state theory, J. Geophys. Res., 84, 2755, 1979.

Southwood, D. J. and Kivelson, M. G.: Mirror instability: 1. Physical Mechanism of linear instability, J. Geophys. Res., 98, 9181, 1993.

Summers, D. and Thorne, R. M.: Plasma microinstabilities driven by loss-cone distributions, J. Plasma Physics, 53, 293, 1995.

Tajiri, M.: Propagation of hydromagnetic waves in collisionless plasmas, II, Kinetic approach, J. Phys. Soc. Jpn., 22, 1482, 1967.

Treumann, R. A.: Kinetic theoretical foundation of Lorentzian statistical mechanics, Physica Scripta, 59, 19, 1999a.

Treumann, R. A.: Generalized Lorentzian thermodynamics, Physica Scripta, 59, 204, 1999b.

Tsurutani, B. T., Smith, E. J., Anderson, R. R., Ogilvie, K. W., Scudder, J. D., Baker, D. N., and Bame, S. J.: Lion roars and nonoscillatory drift mirror waves in the magnetosheath, J. Geophys. Res., 87, 6060, 1982.

Tsurutani, B. T., Southwood, D. J., Smith, E. J., and Balogh, A.: A survey of low frequency waves at Jupiter: The Ulysses encounter, J. Geophys. Res., 98, 21 203, 1993.

Vasyliunas, V. M.: A survey of low-energy electrons in the evening sector of the magnetosphere with OGO 1 and OGO 3, J. Geophys. Res., 73, 2839, 1968.

Williams, D. J. and Mauk, B.: Pitch angle diffusion at Jupiter's moon Ganymede, J. Geophys. Res., 102, 24 283-24 287, 1997. 